\title{
Symptomatic central nervous system involvement in adult patients with acute myeloid leukemia
}

This article was published in the following Dove Press journal:

Cancer Management and Research

29 March 2017

Number of times this article has been viewed

\section{Nael Alakel ${ }^{1, *}$ \\ Friedrich Stölzel ${ }^{1, *}$ \\ Brigitte Mohr' \\ Michael Kramer' \\ Uta Oelschlägel' \\ Christoph Röllig' \\ Martin Bornhäuser' \\ Gerhard Ehninger' \\ Markus Schaich ${ }^{2}$ \\ 'Department of Internal Medicine I, University Hospital Carl Gustav Carus at the Technische Universitaet \\ Dresden, Dresden, ${ }^{2}$ Hematology, \\ Oncology and Palliative Medicine, \\ Rems-Murr-Klinikum, Winnenden, \\ Germany}

*These authors contributed equally to this work
Correspondence: Nael Alakel

Medizinische Klinik und Poliklinik

I, Universitätsklinikum Carl Gustav

Carus Dresden, Fetscherstr. 74, 01307

Dresden, Germany

Tel +493514582321

Fax +49 35I 4585344

Email nael.alakel@uniklinikum-dresden.de

Introduction: Acute myeloid leukemia (AML) rarely involves the central nervous system (CNS). Little is known about the clinical course in adult AML patients since most studies examined pediatric patients. Therefore, this study analyzed the data of patients treated in three prospective trials of the "Study Alliance Leukemia" (SAL) study group for CNS involvement. Methods: In all, 3,261 AML patients included in the prospective AML96, AML2003, and AML60+ trials of the SAL study group were analyzed. Symptomatic patients underwent cerebrospinal fluid (CSF) puncture and CNS involvement was diagnosed depending on morphology and/or flow cytometry of the CSF. Cytogenetic, molecular, clinical, and laboratory parameters were analyzed in order to identify risk factors.

Results: A total of 55 patients had proven symptomatic CNS involvement. Significantly more patients revealed CNS involvement at relapse (34 patients, 2.9\%) compared with first diagnosis (21 patients, $0.6 \%), p<0.001$. CNS involvement at initial diagnosis had a significantly higher frequency in patients with complex aberrant karyotypes, high serum lactate dehydrogenase activity, French-American-British M5 subtype, FLT3-internal tandem duplication (ITD) mutations alone, and co-occurrence of a FLT3-ITD and NPM1 mutation. Furthermore, AML patients with CNS involvement at diagnosis had an inferior outcome compared with patients without CNS involvement even if treated with intrathecal chemotherapy with an overall survival of $11 \%$ versus $30 \%$ at 5 years, $p=0.004$.

Conclusion: This study analyzed the largest data set of adult AML patients with proven CNS involvement reported so far. The data demonstrated very low prevalence of CNS involvement at initial diagnosis in adult patients with AML, and described new risk factors. In patients with risk factors, intense diagnostic and treatment strategies should be employed in the future.

Keywords: Meningeal leukemia, CNS-involvement, cerebrospinal fluid, extramedullary leukemia

\section{Introduction}

In contrast to acute lymphoblastic leukemia, acute myeloid leukemia (AML) rarely involves the central nervous system (CNS) in adult patients while it is more common in pediatric AML patients. ${ }^{1,2}$ Therefore, routine diagnostic evaluation or prophylactic $\mathrm{CNS}$ therapy in adult AML is not performed, although some authors recommend a routine evaluation when hyperleukocytosis is present at diagnosis. ${ }^{3,4}$ Bojsen-Moller and Nielsen performed an autopsy study and found leukemic CNS infiltrates in 46\% of all AML patients, which could point to higher rates of asymptomatic CNS involvement, but might be biased since the remission status of the studied patients was not approximated, and an autopsy study usually includes more patients with unfavorable and relapsed disease. ${ }^{5}$ However, symptoms of CNS involvement may develop later during disease progression or at relapse, which might influence the outcome. Furthermore, CNS involvement might 
be diagnosed through the appearance of either leukemic blasts in the cerebrospinal fluid (CSF) and/or with intracerebral myeloid sarcoma or meningeal AML. Risk factors identified so far include high initial white blood cell (WBC) count in the peripheral blood and AML M5 French-American-British (FAB) morphology. ${ }^{6}$ Several treatment options such as cranial and/or neuro-axis irradiation and intrathecal therapy (ITC) with methotrexate (MTX) and/or cytosine arabinoside (Ara-C) and glucocorticoids along with systemic chemotherapy are widely accepted..$^{7-10}$ Little is known regarding CNS involvement in adult AML and its impact on survival since most studies examined pediatric patients or only a limited number of patients..$^{811-15}$ Therefore, the present study analyzed CNS involvement in patients with AML treated in the prospective AML96, AML2003, and AML60+ trials of the "Study Alliance Leukemia" (SAL) study group for a better understanding of this rare AML entity and to identify patient populations that are at increased risk for CNS disease. This report represents the largest cohort of AML patients analyzed with respect to CNS involvement to date.

\section{Patients and methods}

Between February 1996 and November 2009, 3,526 adult patients with non-acute promyelocytic leukemia AML were included in the prospective AML96, AML2003, and AML60+ trials of the SAL. The studies were approved by the ethics committees of the University of Dresden and all other participating centers. A list of participating centers and ethics committees can be found in the Supplementary material. The protocols were in agreement with the Helsinki declaration and registered with NCT numbers 00180115 (AML96), 00180102 (AML2003), and 00180167 (AML60+). Written informed consent was obtained from all patients. Data were collected and certified by the SAL Data Center.

In the AML96 protocol, patients $<60$ years of age were treated with double induction chemotherapy including standard- and intermediate-dose mitoxantrone, Ara-C, etoposide, and amsacrine and stratified post-remission therapy in different cytogenetic risk groups. Intermediate risk patients with a human leukocyte antigen (HLA)-identical sibling donor were referred to allogeneic hematopoietic stem cell transplant (HSCT). High risk patients were referred to related or unrelated HLA-compatible allogeneic HSCT. ${ }^{16}$ Patients without donors were randomized to intermediate-dose or high-dose Ara-C and mitoxantrone and subsequent autologous HSCT. Patients $>60$ years of age received double induction chemotherapy with daunorubicin and standard-dose Ara-C followed by consolidation therapy consisting of intermediate-dose Ara-C and amsacrine. ${ }^{17}$
In the AML60+ trial, patients $>60$ years of age were included and randomized between the treatment arm of the AML96 study for elderly patients and induction with intermediate-dose Ara-C and mitoxantrone. Allogeneic HSCT was optional for fit patients. ${ }^{18}$

The AML2003 trial included patients below the age of 61 years. Patients were randomized up-front in a two-by-two factorial design with the two factors high-dose Ara-C alone versus high-dose Ara-C plus mitoxantrone and amsacrine for consolidation and standard versus risk adapted intensified consolidation including early allogeneic HSCT during induction therapy and autologous HSCT. All patients received a 3+7 regimen with daunorubicin and Ara-C as induction chemotherapy.

In the presence of neurologic or psychiatric abnormalities, lumbar puncture was performed to confirm or exclude CNS involvement through microscopy and/or flow cytometry of the CSF in all three prospective trials. CNS disease was treated with ITC with MTX, Ara-C, and glucocorticoids. Treatment response and treatment outcome were defined according to the recommended consensus criteria. ${ }^{19}$ Data of proven extramedullary manifestations were available in 3,261 patients, which were included in this study. The patient characteristics are summarized in Table 1. The distributions of cytogenetic, molecular, clinical, and laboratory parameters were compared in order to identify risk factors for CNS involvement. The chi-square test was used for significance testing of comparisons in categorical variables and the Mann-Whitney $U$ test for continuous variables. KaplanMeier method was used to estimate the overall survival (OS). Survival distributions were compared using the log-rank test.

\section{Results}

For this study, all 3,261 patients were analyzed with a median follow-up of 5.1 years. A total of 21 patients had CNS involvement at the time of initial presentation resulting in a prevalence of $0.6 \%$. CNS involvement at AML relapse had a statistically higher incidence of $2.9 \%$ with 34 affected patients among 1,154 patients at relapse versus 21 of 3,261 patients at initial diagnosis $(p<0.010)$.

Characteristics and outcome of CNS disease at initial diagnosis were further analyzed (Table 1). The majority of patients $(n=18,86 \%)$ had de novo AML. The median age of these patients was 54 years (range 22-77 years). Gender and age were equally distributed between patients with CNS involvement and patients without CNS involvement. Extramedullary AML other than CNS was present more frequently in patients with CNS involvement as compared to patients without $\mathrm{CNS}$ involvement $(\mathrm{n}=13,62 \%$ vs $\mathrm{n}=344$, $11 \%, p<0.001)$. 
Table I Comparison of patients' characteristics between AML patients with and without CNS involvement at diagnosis

\begin{tabular}{|c|c|c|c|}
\hline & \multicolumn{2}{|c|}{$\begin{array}{l}\text { AML patients at diagnosis } \\
n=3,26 I\end{array}$} & \multirow[t]{2}{*}{$\overline{p \text {-Value }}$} \\
\hline & $\begin{array}{l}\text { Patients with } \\
\text { CNS involvement } \\
\text { at initial diagnosis } \\
n=2 \text { I }\end{array}$ & $\begin{array}{l}\text { Patients without } \\
\text { CNS involvement } \\
\text { at initial diagnosis } \\
n=3,240\end{array}$ & \\
\hline \multicolumn{4}{|l|}{ Trials } \\
\hline AML96, n & 16 & 1,632 & \\
\hline AML2003, $\mathrm{n}$ & 3 & $\mathrm{I}, 156$ & \\
\hline AML60+, n & 2 & 452 & NA \\
\hline \multicolumn{4}{|l|}{ Gender, n (\%) } \\
\hline Female & $12(57)$ & $1,630(50)$ & 0.496 \\
\hline Male & $9(43)$ & $1,610(50)$ & \\
\hline Median age at diagnosis, years (range) & $54(22-77)$ & $57(15-87)$ & 0.904 \\
\hline Median WBC log mean $\times 10^{9} / \mathrm{L}$ (range)* & $1.13(0.14-2.41)$ & $1.06(0-2.67)$ & 0.054 \\
\hline Median platelets log mean $\times 10^{9} / \mathrm{L}$ (range)* & $1.9(1.04-2.37)$ & $1.72(0-3.12)$ & 0.334 \\
\hline \multicolumn{4}{|l|}{ FAB classification at diagnosis, $n(\%)$} \\
\hline MO & I (5) & $160(5)$ & 0.962 \\
\hline MI & $4(19)$ & $702(22)$ & 0.754 \\
\hline M2 & $5(24)$ & $963(30)$ & 0.536 \\
\hline M4 & $3(14)$ & $509(16)$ & 0.843 \\
\hline M5 & $8(38)$ & $438(14)$ & 0.001 \\
\hline M6 & $0(0)$ & $103(3)$ & 0.404 \\
\hline M7 & $0(0)$ & $22(1)$ & 0.703 \\
\hline Complex aberrant cytogenetic karyotype, n (\%) & $6(29)$ & $398(12)$ & 0.025 \\
\hline \multicolumn{4}{|l|}{ Cytogenetic risk, n (\%) } \\
\hline Adverse & $7(33)$ & $695(22)$ & 0.239 \\
\hline Intermediate & $14(67)$ & $2,331(72)$ & \\
\hline Favorable & $0(0)$ & $214(7)$ & \\
\hline LDH log U/L (range)* & $2.81(2.16-3.86)$ & $2.56(0.38-4.22)$ & 0.008 \\
\hline Extramedullary AML other than CNS, $n$ (\%) & $13(62)$ & $344(\mathrm{II})$ & 0.001 \\
\hline FLT3-ITD positive, $\mathrm{n}(\%)$ & $8(44)$ & $6 I I(2 I)$ & 0.017 \\
\hline NPM I mut, $\mathrm{n}(\%)$ & $8(50)$ & $834(30)$ & 0.078 \\
\hline \multicolumn{4}{|l|}{ FLT3-ITD/NPMI, n (\%) } \\
\hline FLT3-ITD neg/NPMI neg & $7(44)$ & $1,668(60)$ & \\
\hline FLT3-ITD neg/NPMI pos & $I(6)$ & $265(10)$ & 0.002 \\
\hline FLT3-ITD pos/NPMI neg & I (6) & $494(18)$ & \\
\hline FLT3-ITD pos/NPMI pos & $7(44)$ & $335(12)$ & \\
\hline Complete remission (including CRi), $n$ (\%) & $10(48)$ & $2,317(72)$ & 0.016 \\
\hline Allogeneic stem cell transplantation, $n(\%)$ & $6(29)$ & $556(17)$ & 0.045 \\
\hline
\end{tabular}

Note: * Calculated as base 10 logarithms.

Abbreviations: AML, acute myeloid leukemia; CNS, central nervous system; LDH, lactate dehydrogenase activity; ITD, internal tandem duplication; FAB, French-AmericanBritish; NA, not applicable; pos, positive; neg, negative; mut, mutation; WBC, white blood cell.

Significantly more patients with CNS involvement had FLT3-internal tandem duplication (ITD) mutations ( $\mathrm{n}=8$, $44 \%$ vs $\mathrm{n}=611,21 \%, p=0.017$ ) and when grouping FLT3ITD and NPM1 mutations, there was a statistically significant higher occurrence of combined FLT3-ITD and NPM1 mutations in patients with CNS involvement as compared to those without CNS involvement ( $\mathrm{n}=7,44 \%$ vs $\mathrm{n}=335,12 \%$, $p=0.002$ ). Furthermore, patients with CNS involvement exhibited significantly higher levels of lactate dehydrogenase activity (LDH) $(\log \mathrm{U} / 12.81$ vs $2.59, p=0.023$ ) (base 10 logarithm), higher frequencies of FAB M5 morphology $(\mathrm{n}=8,38 \%$ vs $\mathrm{n}=417,13 \%, p=0.001)$ compared with patients without CNS involvement. When analyzing patients at initial diagnosis, complex aberrant karyotypes were significantly more frequent in patients with CNS involvement compared with those without CNS involvement ( $\mathrm{n}=6,29 \%$ vs $\mathrm{n}=398$, $12 \%, p=0.025$ ).

Fourteen of 21 patients with CNS involvement at initial diagnosis were treated with ITC. Survival and complete remission rates for these patients were analyzed. Patients with CNS involvement reached complete remission less frequently than patients without CNS involvement $(\mathrm{n}=10$, $48 \%$ vs $n=2,317,72 \%, p=0.016$ ), and had a significantly inferior outcome even if treated with ITC with an OS of $11 \%$ (95\% confidence interval $[\mathrm{CI}], 0 \%-25 \%$ ) versus $30 \%(95 \%$ CI, $28 \%-32 \%$ ) at 5 years, $p=0.004$ (Figure 1). 


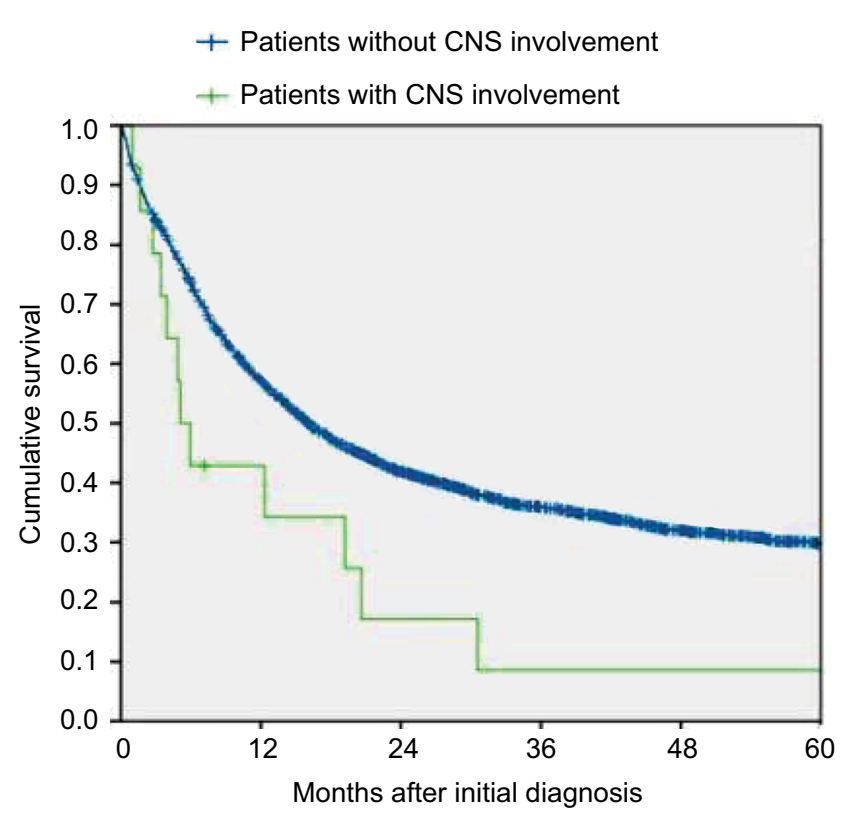

Figure I Overall survival of treated patients with CNS involvement at initial diagnosis of $A M L(n=2 I)$ and patients without CNS involvement at initial diagnosis of AML $(n=3,240)$ within the SAL trials. Log-rank Mantel-Cox $p=0.029$.

Abbreviations: AML, acute myeloid leukemia; CNS, central nervous system; SAL, Study Alliance Leukemia.

\section{Discussion}

AML rarely involves the CNS in adults and there are only a few published studies with controversial results. ${ }^{9,20,21} \mathrm{CNS}$ involvement may remain asymptomatic. Diagnostic lumbar puncture or prophylactic ITC is not routinely recommended without CNS symptoms. Systemic therapy for isolated CNS involvement in parallel to cranial irradiation and/or ITC seems to be necessary in order to avoid marrow relapse. ${ }^{22,23}$

This study analyzes the largest data set of adult AML patients with proven CNS involvement reported so far. An overall prevalence of $0.6 \%$ was found at initial diagnosis which is lower than previously reported prevalence ranging between $2 \%$ and $8 \%{ }^{1,24,25}$ One possible reason of higher rates in the earlier reports could be the fact that some of these studies were done in pediatric patients while others included promyelocytic leukemia, used imaging studies with magnetic resonance imaging instead of lumbar puncture, or included patients at relapse as well. ${ }^{24-28}$ However, some of these imaging data might be conflicting since they possibly rather represent intracerebral myeloid sarcoma, meningeal AML, or simply inflammatory enhancement. Furthermore, the data from the present analysis reflect only the patients in whom manifestation of CNS involvement was clinically apparent due to neurological and/or psychiatric symptoms. Thus, this could implicate that the real prevalence including asymptomatic CNS involvement is higher, although another study suggested that this might not be the case. ${ }^{29}$

In this analysis, symptomatic CNS involvement was frequently accompanied by other extramedullary AML manifestations and higher prevalence of FLT3-ITD mutations, whereas NPM1 mutations alone had no significant higher incidence in these patients. As previously shown, FAB M5 morphology, complex aberrant karyotype, higher LDH levels, and higher WBC count at diagnosis represent risk factors for CNS involvement. ${ }^{3,8,13,25}$ Interestingly, other studies showed an association of CNS involvement in AML with certain cytogenetic abnormalities such as inv(16), del(5q), del(7q), trisomy of chromosome $8, \mathrm{t}(8 ; 21)$, and abnormalities of chromosome 11q23, which could not be confirmed for patients with CNS involvement at the initial diagnosis of AML..$^{25,30,31}$

However, upon extending the data analysis by including also CNS involvement of AML at relapse, statistically significant higher incidence of CNS involvement was confirmed for patients with trisomy of chromosome 8 , trisomy of chromosome $22, \mathrm{t}(9 ; 11), \mathrm{t}(6 ; 11)$, and complex karyotype. The study could not confirm a higher prevalence for patients with $t(8 ; 21)$, inv(16), monosomy of chromosome 5 , or monosomy of chromosome 7 (Table 2). In total, abnormalities of chromosome 11q23 were evident in $20 \%$ of all AML patients with CNS involvement either at initial diagnosis or at relapse of CNS involvement ( $n=11 / 55)$. In 7 patients 11q23 abnormalities were only detected using FISH analysis (Fluorescence in situ hybridization). In these cases the 11q23 translocation was cryptic by karyotyping. This reflects a higher prevalence of $11 \mathrm{q} 23$ abnormalities than the estimated $6 \%$ in AML with a higher occurrence in myelomonocytic AML. ${ }^{32,33}$ Hence, data from the present analysis confirm the previously reported higher occurrence of 11q23 abnormalities of patients with CNS disease as reported by others recently. ${ }^{13,25,34}$

The impact of CNS involvement on long-term treatment outcome has been discussed controversially. ${ }^{4,8,10,21}$ Recently, Shihadeh et al suggested that adult AML patients with CNS involvement have poor OS. ${ }^{25}$ The present study could confirm this finding based on the largest number of patients with CNS involvement and define further risk factors (FLT3-ITD mutations, complex aberrant karyotypes) that might help to identify AML patients at risk for CNS involvement (Figure 2). However, due to the low frequency and low ITC chemotherapy-initiation at diagnosis in the three reported AML trials, differently sized patient cohorts have been compared and the statistical impact must be interpreted carefully. In summary, this analysis suggests a very low frequency of symptomatic CNS AML involvement in adults. Because of the obviously poor prognosis of CNS involvement, screening for risk factors of CNS involvement at diagnosis in symptomatic AML patients with suspicion for CNS involvement may be useful to identify a patient population benefiting from intensified treatment strategies in the future. 
Table 2 Comparison of cytogenetic profile between AML patients with and without CNS involvement at diagnosis or relapse

\begin{tabular}{|c|c|c|c|}
\hline \multirow[t]{2}{*}{$\begin{array}{l}\text { Cytogenetic } \\
\text { profile }\end{array}$} & \multicolumn{2}{|c|}{$\begin{array}{l}\text { AML patients at diagnosis } \\
n=3,26 I\end{array}$} & \multirow[t]{2}{*}{ p-Value } \\
\hline & $\begin{array}{l}\text { Patients with } \\
\text { CNS involvement } \\
\text { at initial diagnosis } \\
\text { and relapse } \\
n=55\end{array}$ & $\begin{array}{l}\text { Patients } \\
\text { without CNS } \\
\text { involvement } \\
\text { at any time } \\
n=3,206\end{array}$ & \\
\hline $\begin{array}{l}\text { Normal karyotype, } \\
\text { n (\%) }\end{array}$ & $16(31)$ & $\mathrm{I}, 472(50)$ & 0.158 \\
\hline Trisomy 8, n (\%) & $10(21)$ & $254(8)$ & 0.001 \\
\hline Trisomy 22, n (\%) & $3(6)$ & $62(2)$ & 0.034 \\
\hline Trisomy II, n (\%) & I (2) & $35(I)$ & 0.514 \\
\hline Trisomy I3, n (\%) & $I(2)$ & $42(I)$ & $0.64 I$ \\
\hline Trisomy 2I, n (\%) & $2(4)$ & $69(2)$ & 0.343 \\
\hline Monosomy 5, n (\%) & $0(0)$ & $26(I)$ & 0.531 \\
\hline Monosomy 7, n (\%) & $0(0)$ & I 44 (4) & 0.133 \\
\hline $\mathrm{t}(3 ; 3), \mathrm{n}(\%)$ & $0(0)$ & $8(1)$ & 0.729 \\
\hline $\mathrm{t}(\mathrm{I} ; 7), \mathrm{n}(\%)$ & $0(0)$ & I (0) & 0.903 \\
\hline t(6;।I), n (\%) & I (2) & $6(0)$ & 0.005 \\
\hline $\mathrm{t}(8 ; 2 \mathrm{I}), \mathrm{n}(\%)$ & $2(4)$ & $128(4)$ & 0.951 \\
\hline $\mathrm{t}(9 ; \mathrm{II}), \mathrm{n}(\%)$ & $3(6)$ & $4 I(I)$ & 0.003 \\
\hline $\mathrm{t}(6 ; 9), \mathrm{n}(\%)$ & $0(0)$ & $22(I)$ & 0.565 \\
\hline $\mathrm{t}(9 ; 22), \mathrm{n}(\%)$ & $0(0)$ & $7(\mathrm{I})$ & 0.746 \\
\hline t(II;19), n (\%) & $0(0)$ & $16(1)$ & 0.624 \\
\hline $\operatorname{inv}(16), n(\%)$ & $3(6)$ & $150(5)$ & 0.610 \\
\hline $\operatorname{lnv}(3)(q 2 \mid q 26)$ & $0(0)$ & $20(I)$ & 0.583 \\
\hline
\end{tabular}

Abbreviations: AML, acute myeloid leukemia; CNS, central nervous system.

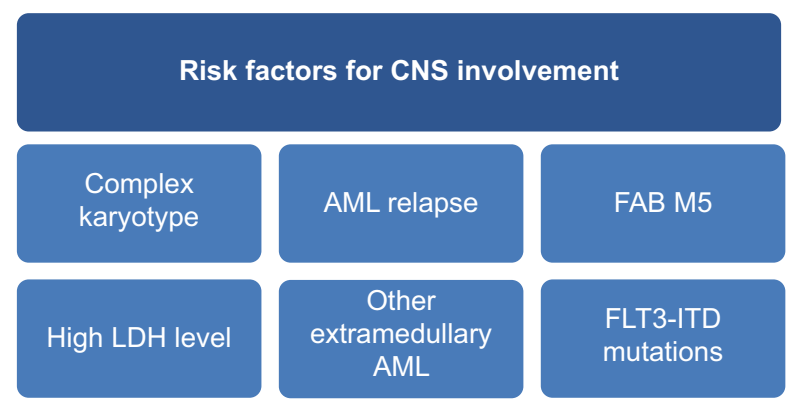

Figure 2 Risk factors for symptomatic AML patients with potential CNS involvement at initial diagnosis of AML.

Abbreviations: AML, acute myeloid leukemia; CNS, central nervous system; LDH, lactate dehydrogenase activity; ITD, internal tandem duplication; FAB, FrenchAmerican-British.

\section{Acknowledgments}

The contribution of all patients and physicians in the various trials of the German SAL is highly appreciated. This study was presented in part at the 17th Annual Congress of the European Hematology Association, June 14-17, 2012, Amsterdam, the Netherlands.

\section{Disclosure}

The authors report no conflicts of interests in this work.

\section{References}

1. Abbott BL, Rubnitz JE, Tong X, et al. Clinical significance of central nervous system involvement at diagnosis of pediatric acute myeloid leukemia: a single institution's experience. Leukemia. 2003;17(11):2090-2096.

2. Rees JK, Gray RG, Swirsky D, Hayhoe FG. Principal results of the Medical Research Council's 8th acute myeloid leukaemia trial. Lancet (London, England). 1986;2(8518):1236-1241.

3. Cassileth PA, Sylvester LS, Bennett JM, Begg CB. High peripheral blast count in adult acute myelogenous leukemia is a primary risk factor for CNS leukemia. J Clin Oncol. 1988;6(3):495-498.

4. Döhner H, Estey EH, Amadori S, et al. Diagnosis and management of acute myeloid leukemia in adults: Recommendations from an international expert panel, on behalf of the European LeukemiaNet. Blood. 2010; 115(3):453-474.

5. Bojsen-Møller M, Nielsen JL. CNS involvement in leukaemia. An autopsy study of 100 consecutive patients. Acta Pathol Microbiol Immunol Scand A. 1983;91(4):209-216.

6. Stewart DJ, Keating MJ, McCredie KB, et al. Natural history of central nervous system acute leukemia in adults. Cancer. 1981;47(1):184-196.

7. Bakst RL, Tallman MS, Douer D, Yahalom J. How I treat extramedullary acute myeloid leukemia. Blood. 2011;118(14):3785-3793.

8. Castagnola C, Nozza A, Corso A, Bernasconi C. The value of combination therapy in adult acute myeloid leukemia with central nervous system involvement. Haematologica. 1997;82(5):577-580.

9. Mayadev JS, Douglas JG, Storer BE, Appelbaum FR, Storb R. Impact of cranial irradiation added to intrathecal conditioning in hematopoietic cell transplantation in adult acute myeloid leukemia with central nervous system involvement. Int J Radiat Oncol Biol Phys. 2011;80(1):193-198.

10. Sanders KE, Ha CS, Cortes-Franco JE, Koller CA, Kantarjian HM, Cox JD. The role of craniospinal irradiation in adults with a central nervous system recurrence of leukemia. Cancer. 2004;100(10):2176-2180.

11. Bommer M, von Harsdorf S, Döhner H, Bunjes D, Ringhoffer M. Neoplastic meningitis in patients with acute myeloid leukemia scheduled for allogeneic hematopoietic stem cell transplantation. Haematologica. 2010;95(11):1962-1972.

12. Frick J, Titch PS, Hansen RM, et al. Successful treatment of meningeal leukemia using systemic high dose cytosine arabinoside. J Clin Oncol. 1984;2:365-368.

13. Johnston DL, Alonzo TA, Gerbing RB, Lange BJ, Woods WG. Risk factors and therapy for isolated central nervous system relapse of pediatric acute myeloid leukemia. J Clin Oncol. 2005;23(36):9172-9178.

14. Kobayashi R, Tawa A, Hanada R, et al. Extramedullary infiltration at diagnosis and prognosis in children with acute myelogenous leukemia. Pediatr Blood Cancer. 2007;48(4):393-398.

15. Pui $\mathrm{CH}$, Howard SC. Current management and challenges of malignant disease in the CNS in paediatric leukaemia. Lancet Oncol. 2008; 9(3):257-268.

16. Schaich M, Röllig C, Soucek S, et al. Cytarabine dose of $36 \mathrm{~g} / \mathrm{m}^{2}$ compared with $12 \mathrm{~g} / \mathrm{m}^{2}$ within first consolidation in acute myeloid leukemia: results of patients enrolled onto the prospective randomized AML96 study. J Clin Oncol. 2011;29(19):2696-2702.

17. Röllig C, Thiede C, Gramatzki M, et al. A novel prognostic model in elderly patients with acute myeloid leukemia: results of 909 patients entered into the prospective AML96 trial. Blood. 2010;116(6):971-978.

18. Röllig C, Kramer M, Hanel M, et al. Induction treatment in elderly patients with acute myeloid leukemia (AML): randomized comparison of intermediate-dose cytarabine plus mitoxantrone (IMA) versus standard-dose cytarabine plus daunorubicin (DA) in $492 \mathrm{aml}$ patients $>60$ years - results. Blood. 2015;116(21):334.

19. Cheson BD, Bennett JM, Kopecky KJ, et al. Revised recommendations of the International Working Group for Diagnosis, Standardization of Response Criteria, Treatment Outcomes, and Reporting Standards for Therapeutic Trials in Acute Myeloid Leukemia. J Clin Oncol. 2003; 21(24):4642-4649.

20. Oshima K, Kanda Y, Yamashita T, et al. Central nervous system relapse of leukemia after allogeneic hematopoietic stem cell transplantation. Biol Blood Marrow Transplant. 2008;14(10):1100-1107. 
21. Woods WG, Kobrinsky N, Buckley J, et al. Intensively timed induction therapy followed by autologous or allogeneic bone marrow transplantation for children with acute myeloid leukemia or myelodysplastic syndrome: a Childrens Cancer Group pilot study. J Clin Oncol. 1993;11(8): 1448-1457.

22. Simone J V. Treatment of meningeal leukemia. J Clin Oncol. 1984; 2(5):357-358

23. Steinherz P, Jereb B, Galicich J. Therapy of CNS leukemia with intraventricular chemotherapy and low-dose neuraxis radiotherapy. J Clin Oncol. 1985;3(9):1217-1226.

24. Grier HE, Gelber RD, Camitta BM, et al. Prognostic factors in childhood acute myelogenous leukemia. J Clin Oncol. 1987;5(7):1026-1032.

25. Shihadeh F, Reed V, Faderl S, et al. Cytogenetic profile of patients with acute myeloid leukemia and central nervous system disease. Cancer. 2012;118(1):112-117.

26. Martínez-Cuadrón D, Montesinos P, Pérez-sirvent M, et al. Central nervous system involvement at first relapse in patients with acute myeloid leukemia. Haematologica. 2011;96(9):1375-1379.

27. de Botton S, Sanz MA, Chevret S, et al. Extramedullary relapse in acute promyelocytic leukemia treated with all-trans retinoic acid and chemotherapy. Leukemia. 2006;20(1):35-41.

28. Specchia G, Lo Coco F, Vignetti M, et al. Extramedullary involvement at relapse in acute promyelocytic leukemia patients treated or not with all-trans retinoic acid: a report by the Gruppo Italiano Malattie Ematologiche dell'Adulto. J Clin Oncol. 2001;19(20):4023-4028.
29. Morrison FS, Kopecky KJ, Head DR, et al. Late intensification with POMP chemotherapy prolongs survival in acute myelogenous leukemia-results of a Southwest Oncology Group study of rubidazone versus adriamycin for remission induction, prophylactic intrathecal therapy, late intensification, and 1. Leukemia. 1992;6(7):708-714.

30. Holmes R, Keating MJ, Cork A, et al. A unique pattern of central nervous system leukemia in acute myelomonocytic leukemia associated with inv(16)(p13q22). Blood. 1985;65(5):1071-1078.

31. Ghaddar HM, Plunkett W, Kantarjian HM, et al. Long-term results following treatment of newly-diagnosed acute myelogenous leukemia with continuous-infusion high-dose cytosine arabinoside. Leukemia. 1994;8(8):1269-1274.

32. Grimwade D, Hills RK, Moorman AV, et al. Refinement of cytogenetic classification in acute myeloid leukaemia: determination of prognostic significance of rarer recurring chromosomal abnormalities amongst 5,876 younger adult patients treated in the UK Medical Research Council trials. Br J Haematol. 2010; 116:354-365.

33. Sorensen PHB, Chen CS, Smith FO, et al. Molecular rearrangements of the MLL gene are present in most cases of infant acute myeloid leukemia and are strongly correlated with monocytic or myelomonocytic phenotypes. J Clin Invest. 1994;93(1):429-437.

34. Chang H, Brandwein J, Yi Q-L, Chun K, Patterson B, Brien B. Extramedullary infiltrates of AML are associated with CD56 expression, 11q23 abnormalities and inferior clinical outcome. Leuk Res. 2004;28(10): 1007-1011.
Cancer Management and Research

\section{Publish your work in this journal}

Cancer Management and Research is an international, peer-reviewed open access journal focusing on cancer research and the optimal use of preventative and integrated treatment interventions to achieve improved outcomes, enhanced survival and quality of life for the cancer patient. The manuscript management system is completely online and includes
Dovepress

a very quick and fair peer-review system, which is all easy to use. Visi $\mathrm{http}: / / \mathrm{www}$.dovepress.com/testimonials.php to read real quotes from published authors. 